\title{
Dynamic integration of process planning and scheduling using a discrete particle swarm optimization algorithm
}

\author{
Yu, M.R. ${ }^{a}{ }^{*}$, Yang, B. ${ }^{a}$, Chen, Y. ${ }^{b}$ \\ ${ }^{a}$ School of Mechatronic Engineering, North University of China, Taiyuan, P.R. China \\ ${ }^{\mathrm{b}}$ North Automatic Control Technology Institute, Taiyuan, P.R. China
}

\begin{abstract}
A B S T R A C T
Because of the inherent relationship between process planning and scheduling, integration of process planning and scheduling (IPPS) provides a new path for further improvements of these two activities. Therefore, a novel twophase IPPS approach is put forward in this paper. In the new method, the preplanning phase generates a process network for each job with consideration of the static shop floor status. After that, the final planning phase simultaneously creates the process plan of each job and the scheduling plan according to the current shop floor status. Based on the modified definition of IPPS and the proposed mathematical model, the IPPS problem and the dynamic IPPS problem can be solved together. Furthermore, a discrete particle swarm optimization (DPSO) algorithm is proposed to solve the IPPS optimization problem. In the DPSO algorithm, the particles update their positions by crossing with their own historical best positions (pbests) and the global best position of the population (gbest). In order to avoid local convergence, an external archive is introduced to keep more than one elite, and the gbest of each particle is randomly selected from the external archive. Furthermore, mutation operation is introduced to enhance the local search ability of DPSO algorithm. Finally, some comparative results are given to verify the efficiency and effectiveness of the proposed IPPS method and the DPSO algorithm as well as the dynamic IPPS method.
\end{abstract}

\begin{tabular}{l} 
A R T I C L E I N F O \\
\hline Keywords: \\
Process planning; \\
Scheduling; \\
Dynamic integration; \\
Mathematical model; \\
Optimization; \\
Discrete particle swarm optimiza- \\
tion (DPSO) \\
*Corresponding author: \\
yumingrang@163.com \\
(Yu, M.R.) \\
Article history: \\
Received 4 December 2017 \\
Revised 21 August 2018 \\
Accepted 24 August 2018
\end{tabular}

(C) 2018 CPE, University of Maribor. All rights reserved.

\section{References}

[1] Phanden, R.K., Jain, A., Verma, R. (2011). Integration of process planning and scheduling: A state-of-the-art review, International Journal of Computer Integrated Manufacturing, Vol. 24, No. 6, 517-534, doi: 10.1080/09511 92x.2011.562543.

[2] Chryssolouris, G., Chan, S., Cobb, W. (1984). Decision making on the factory floor: An integrated approach to process planning and scheduling, Robotics and Computer-Integrated Manufacturing, Vol. 1, No. 3-4, 315-319, doi: 10.1016/0736-5845(84)90020-6.

[3] Yu, M., Zhang, Y., Chen, K., Zhang, D. (2015). Integration of process planning and scheduling using a hybrid GA/PSO algorithm, The International Journal of Advanced Manufacturing Technology, Vol. 78, No. 1-4, 583-592, doi: 10.1007/s00170-014-6669-7.

[4] Tan, W., Khoshnevis, B. (2000). Integration of process planning and scheduling - A review, Journal of Intelligent Manufacturing, Vol. 11, No. 1, 51-63, doi: 10.1023/a:1008952024606.

[5] Li, X., Gao, L., Zhang, C., Shao, X. (2010). A review on integrated process planning and scheduling, International Journal of Manufacturing Research, Vol. 5, No. 2, 161-180, doi: 10.1504/IJMR.2010.031630.

[6] Zhang, H., Liu, S., Moraca, S., Ojstersek, R. (2017). An effective use of hybrid metaheuristics algorithm for job shop scheduling problem, International Journal of Simulation Modelling, Vol. 16, No. 4, 644-657, doi: $\underline{10.2507 / I J S I M M ~ 16(4) 7.400 . ~}$ 
[7] Huang, X.W., Zhao, X.Y., Ma, X.L. (2014). An improved genetic algorithm for job-shop scheduling problem with process sequence flexibility, International Journal of Simulation Modelling, Vol. 13, No. 4, 510-522, doi: 10.2507/ IJSIMM13(4)CO20.

[8] Guo, Y.W., Li, W.D., Mileham, A.R., Owen, G.W. (2009). Optimisation of integrated process planning and scheduling using a particle swarm optimisation approach, International Journal of Production Research, Vol. 47, No. 14, 3775-3796, doi: 10.1080/00207540701827905.

[9] Leung, C.W., Wong, T.N., Mak, K.L., Fung, R.Y.K. (2010). Integrated process planning and scheduling by an agentbased ant colony optimization, Computers \& Industrial Engineering, Vol. 59, No. 1, 166-180, doi: 10.1016/j.cie. 2009.09.003.

[10] Zhang, H.-C., Merchant, M.E. (1993). IPPM - A prototype to integrate process planning and job shop scheduling functions, CIRP Annals, Vol. 42, No. 1, 513-518, doi: 10.1016/S0007-8506(07)62498-6.

[11] Dai, M., Tang, D., Xu, Y., Li, W. (2014). Energy-aware integrated process planning and scheduling for job shops, Proceedings of the Institution of Mechanical Engineers, Part B: Journal of Engineering Manufacture, Vol. 229, No. 1, 13-26, doi: $10.1177 / 0954405414553069$.

[12] Galzina, V., Lujić, R., Šarić, T. (2012). Adaptive fuzzy particle swarm optimization for flow-shop scheduling problem, Tehnički vjesnik - Tehnical Gazette, Vol. 19, No. 1, 151-157.

[13] Chaudhry, I.A., Usman, M. (2017). Integrated process planning and scheduling using genetic algorithms, Tehnički vjesnik - Technical Gazette, Vol. 24, No. 5, 1401-1409, doi: 10.17559/TV-20151121212910.

[14] Zhang, L., Wong, T.N. (2015). An object-coding genetic algorithm for integrated process planning and scheduling, European Journal of Operational Research, Vol. 244, No. 2, 434-444, doi: 10.1016/i.ejor.2015.01.032.

[15] Wang, S., Lu, X., Li, X.X., Li, W.D. (2015). A systematic approach of process planning and scheduling optimization for sustainable machining, Journal of Cleaner Production, Vol. 87, No. 914-929, doi: 10.1016/i.jclepro.2014. $\underline{10.008}$.

[16] Kim, Y.K., Park, K., Ko, J. (2003). A symbiotic evolutionary algorithm for the integration of process planning and job shop scheduling, Computers \& Operations Research, Vol. 30, No. 8, 1151-1171, doi: 10.1016/S0305-0548(02) 00063-1.

[17] Ho, Y.-C., Moodie, C.L. (1996). Solving cell formation problems in a manufacturing environment with flexible processing and routeing capabilities, International Journal of Production Research, Vol. 34, No. 10, 2901-2923, doi: $10.1080 / 00207549608905065$.

[18] Guo, Y.W., Li, W.D., Mileham, A.R., Owen, G.W. (2009). Applications of particle swarm optimisation in integrated process planning and scheduling, Robotics and Computer-Integrated Manufacturing, Vol. 25, No. 2, 280-288, doi: 10.1016/j.rcim.2007.12.002.

[19] Li, X., Gao, L., Shao, X., Zhang, C., Wang, C. (2010). Mathematical modeling and evolutionary algorithm-based approach for integrated process planning and scheduling, Computers \& Operations Research, Vol. 37, No. 4, 656667, doi: 10.1016/j.cor.2009.06.008.

[20] Kennedy, J., Eberhart, R. (1995). Particle swarm optimization, In: Proceedings of ICNN'95 - International Conference on Neural Networks, Perth, Australia, 1942-1948, doi: 10.1109/ICNN.1995.488968.

[21] Lin, T.-L., Horng, S.-J., Kao, T.-W., Chen, Y.-H., Run, R.-S., Chen, R.-J., Lai, J.-L., Kuo, I.-H. (2010). An efficient jobshop scheduling algorithm based on particle swarm optimization, Expert Systems with Applications, Vol. 37, No. 3, 2629-2636, doi: 10.1016/i.eswa.2009.08.015.

[22] Sha, D.Y., Hsu, C.-Y. (2006). A hybrid particle swarm optimization for job shop scheduling problem, Computers \& Industrial Engineering, Vol. 51, No. 4, 791-808, doi: 10.1016/j.cie.2006.09.002.

[23] Shi, Y., Eberhart, R. (1998). A modified particle swarm optimizer, In: 1998 IEEE International Conference on Evolutionary Computation Proceedings. IEEE World Congress on Computational Intelligence, Anchorage, USA, 6973, doi: $10.1109 /$ ICEC.1998.699146.

[24] Zhang, G., Gao, L., Shi, Y. (2011). An effective genetic algorithm for the flexible job-shop scheduling problem, Expert Systems with Applications, Vol. 38, No. 4, 3563-3573, doi: 10.1016/j.eswa.2010.08.145.

[25] Li, W.D., Ong, S.K., Nee, A.Y.C. (2004). Optimization of process plans using a constraint-based tabu search approach, International Journal of Production Research, Vol. 42, No. 10, 1955-1985, doi: 10.1080/0020754031 0001652897.

[26] Kacem, I., Hammadi, S., Borne, P. (2002). Pareto-optimality approach for flexible job-shop scheduling problems: Hybridization of evolutionary algorithms and fuzzy logic, Mathematics and Computers in Simulation, Vol. 60, No. 3-5, 245-276, doi: 10.1016/S0378-4754(02)00019-8.

[27] Kacem, I., Hammadi, S., Borne, P. (2002). Approach by localization and multiobjective evolutionary optimization for flexible job-shop scheduling problems, IEEE Transactions on Systems, Man, and Cybernetics, Part C (Applications and Reviews), Vol. 32, No. 1, 1-13, doi: 10.1109/tsmcc.2002.1009117.

[28] Brandimarte, P. (1993). Routing and scheduling in a flexible job shop by tabu search, Annals of Operations Research, Vol. 41, No. 3, 157-183, doi: 10.1007/bf02023073.

[29] Li, J.-Q., Pan, Q.-K., Gao, K.-Z. (2011). Pareto-based discrete artificial bee colony algorithm for multi-objective flexible job shop scheduling problems, The International Journal of Advanced Manufacturing Technology, Vol. 55, No. 9-12, 1159-1169, doi: 10.1007/s00170-010-3140-2.

[30] Wang, X., Gao, L., Zhang, C., Shao, X. (2010). A multi-objective genetic algorithm based on immune and entropy principle for flexible job-shop scheduling problem, The International Journal of Advanced Manufacturing Technology, Vol. 51, No. 5-8, 757-767, doi: 10.1007/s00170-010-2642-2.

[31] Xia, W., Wu, Z. (2005). An effective hybrid optimization approach for multi-objective flexible job-shop scheduling problems, Computers \& Industrial Engineering, Vol. 48, No. 2, 409-425, doi: 10.1016/j.cie.2005.01.018. 
[32] Moslehi, G., Mahnam, M. (2011). A Pareto approach to multi-objective flexible job-shop scheduling problem using particle swarm optimization and local search, International Journal of Production Economics, Vol. 129, No. 1, 14-22, doi: 10.1016/j.ijpe.2010.08.004.

[33] Bagheri, A., Zandieh, M., Mahdavi, I., Yazdani, M. (2010). An artificial immune algorithm for the flexible job-shop scheduling problem, Future Generation Computer Systems, Vol. 26, No. 4, 533-541, doi: 10.1016/i.future.2009. 10.004.

[34] Xing, L.-N., Chen, Y.-W., Yang, K.-W. (2009). An efficient search method for multi-objective flexible job shop scheduling problems, Journal of Intelligent Manufacturing, Vol. 20, No. 3, 283-293, doi: 10.1007/s10845-0080216-z.

[35] He, W., Sun, D.-H. (2013). Scheduling flexible job shop problem subject to machine breakdown with route changing and right-shift strategies, The International Journal of Advanced Manufacturing Technology, Vol. 66, No. 1-4, 501-514, doi: $10.1007 / \mathrm{s} 00170-012-4344-4$. 


\section{APEM}

Advances in Production Engineering \& Management Letnik 13 | Številka 3 | September 2018 | Strani 279-296 https://doi.org/10.14743/apem2018.3.290
ISSN 1854-6250

Spletna stran: apem-journal.org Izvirni znanstveni članek

\title{
Dinamična integracija načrtovanja in terminiranja procesa z uporabo diskretnega optimizacijskega algoritma roja delcev
}

\author{
Yu, M.R. ${ }^{a,}{ }^{*}$, Yang, B. ${ }^{a}$, Chen, Y. ${ }^{b}$ \\ ${ }^{a}$ School of Mechatronic Engineering, North University of China, Taiyuan, P.R. China \\ ${ }^{b}$ North Automatic Control Technology Institute, Taiyuan, P.R. China
}

\section{POVZETEK}

Zaradi neločljive povezave med načrtovanjem in terminiranjem procesov je povezovanje načrtovanja in terminiranja procesov (IPPS) ena izmed poti za izboljšavo teh dveh dejavnosti. Zato $\mathrm{v}$ tem prispevku predstavljamo nov dvostopenjski pristop IPPS. Pri predlagani metodi se $v$ prvi fazi načrtovanja, ob upoštevanju statičnega stanja v proizvodnji, ustvari procesna mreža za vsako opravilo. Nato se v končni fazi načrtovanja hkrati ustvari procesni načrt vsakega delovnega poteka in načrt razporeditve del glede na trenutno stanje proizvodnje. Na podlagi spremenjene definicije IPPS in predlaganega matematičnega modela lahko problem IPPS in dinamični problem IPPS rešimo skupaj. Poleg tega je predlagan diskretnimi algoritem za optimizacijo z rojem delcev (DPSO) za reševanje problema optimizacije IPPS. V algoritmu DPSO delci posodabljajo svoje položaje tako, da križajo svoje lastne zgodovinsko najboljše položaje in najboljši položaj celotne populacije (gbest). Da bi preprečili lokalno konvergenco, smo uvedli zunanjo arhivsko bazo, ki hrani več kot eno elito, gbest vsakega delca pa se naključno izbere iz te baze. Poleg tega smo vpeljali operator mutacije, s čimer se poveča lokalna možnost iskanja algoritma DPSO. Na koncu smo podali nekatere primerjalne rezultate za preverjanje učinkovitosti in uspešnosti predlagane metode IPPS in algoritma DPSO ter dinamične metode IPPS.

\section{PODATKI O ČLANKU}

Ključne besede:

Načrtovanje procesa;

Terminiranje;

Dinamična integracija;

Matematični model;

Optimizacija;

Diskretna optimizacija z rojem

delcev (DPSO)

*Kontaktna oseba: yumingrang@163.com (Yu, M.R.)

Zgodovina članka:

Prejet 4. decembra 2017

Popravljen 21. avgusta 2018

Sprejet 24. avgusta 2018 